\title{
Collaborative Projects: Unleashing Early Career Scientists' Power
}

Pascal Bodmer (1) https://orcid.org/0000-0001-7383-889X, Katrin Attermeyer (ㄴ) https://orcid.org/0000-0002-6503-9497, Ada Pastor 1 https://orcid.org/0000-0002-7114-770X, Núria Catalán $\$ https://orcid.org/0000-0002-2433-4190

DOI

10.1016/i.tree.2019.07.016

Original publication date

28 August 2019 (Available online)

\section{Document version}

Accepted manuscript

\section{Published in}

Trends in Ecology \& Evolution

\section{Citation}

Bodmer P, Attermeyer K, Pastor A, Catalán N. Collaborative Projects: Unleashing Early Career Scientists' Power. Trends in Ecology \& Evolution. 2019;34(10):871-4. 
1 Collaborative projects: Unleashing Early Career Scientists' Power

2

3 Pascal Bodmer ${ }^{\dagger}{ }^{1,2}$, Katrin Attermeyer $*^{3}$, Ada Pastor $*^{4}$, Núria Catalán $*^{5}$

$4 \quad *$ All authors contributed equally to this work; Author order was computed randomly

5

$6 \quad{ }^{1}$ Chemical Analytics and Biogeochemistry, Leibniz-Institute of Freshwater Ecology and Inland

7 Fisheries, Berlin, Germany

$8 \quad 2$ Institute for Environmental Sciences, University of Koblenz-Landau, Landau, Germany

$9 \quad{ }^{3}$ WasserCluster Lunz, 3293 Lunz am See, Austria

$10{ }^{4}$ Department of Bioscience, Aarhus University, Aarhus, Denmark

$11{ }^{5}$ Catalan Institute for Water Research (ICRA), Girona, Spain

12

$13{ }^{\dagger}$ Correspondence: bodmerpascal@gmail.com (P. Bodmer), @BodmerPascal;

14 katrin.attermeyer@wcl.ac.at (K. Attermeyer), @katat85; adapastor@bios.au.dk (A. Pastor),

$15 @$ @apastoroliveras; ncatalangarcia@gmail.com (N. Catalán), @NCatalanGarcia

16

17 Keywords: career development, collaboration, junior researcher, networking, translational

18 ecology

19 
Abstract: Collaborative research projects exclusively targeted to early career researchers (ECRs) have been initiated in Europe. So far, the first two collaborative projects have united more than 80 ECRs. We describe the structure and benefits of such initiatives for the ECRs and highlight the positive influence on the whole scientific community.

Global environmental and ecological questions need to be addressed by the efforts of research collectives [1]. Collaborative and geographically distributed projects require welldeveloped constructed networks, which are often missing at the beginning of a scientific career [2]. The effective involvement of early career researchers (ECRs) in regular collaborative projects often poses major challenges [3]. Large collaborative projects often require more planning and longer turnaround times for publication. This is difficult for ECRs who are often on short term contracts and need relatively quickly produced research outputs for career advancement. Another challenge is that, regardless of who carries out the actual research, credit for research is most often awarded to the most senior project participant [3]. Hence, achieving individual recognition for their work is one of the most critical challenges for ECRs. In recent years, the Iberian Association of Limnology (AlL') and the European Federation for Freshwater Sciences

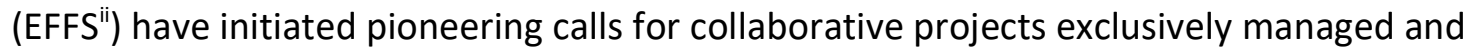
conducted by ECRs [4-6]. The aim of these research calls was not only to boost innovative science [7] but also to provide peer-collaboration platforms among scientists at the beginning of their careers. In particular, the requirements for the research projects were to conduct original research on any aspect of the ecology of inland waters using inexpensive and simple methods and to promote networking among European ECRs. The overall aim of this article is to present

\footnotetext{
i https://www.limnetica.com/

ii http://www.freshwatersciences.eu

Page 2 of 11
} 
these two pioneer initiatives as new instruments for ERCs career development to the scientific community and promote the development of similar ones across different ecological disciplines. More specifically, the objectives were: i) to describe their implementation from local to multinational scales, ii) to advocate the benefits of these initiatives for ECRs and eventually, the scientific community, and iii) to share our experience as leaders of these two pioneer projects by critically describing the main strengths and challenges.

Implementation from local to multinational scales - The two collaborative ECR projects that have been completed to date are the DOMIPEX ${ }^{\mathrm{iii}}$ and the EuroRun ${ }^{\mathrm{iv}}$ projects, initiated by AIL and EFFS, respectively. Two more projects are currently ongoing (Urban Algaev and AGRHYDROM ${ }^{\mathrm{vi}}$ ). All of these projects are in freshwater sciences. Combined, the two completed projects involved 28 teams, 32 research institutions, and 82 researchers from 12 European countries (Fig. 1). From the 82 researchers participating in the pioneer projects DOMIPEX and EuroRun, 60\% were Ph.D. students, $16 \%$ short-term postdocs (i.e. <2 years after Ph.D. completion), and the remaining $24 \%$ were a mix of long-term postdocs, tenure track position holders, and master students. The DOMIPEX and EuroRun projects were composed of 2-7 members per team geographically distributed across Europe (Fig. 1). For DOMIPEX, an organizational meeting open to all participants and members of the AIL association was done to present and discuss the preliminary plan for the project during the AIL 2014 conference. For EuroRun, team representatives joined a workshop, which aimed at building the measuring equipment, training

\footnotetext{
iii https://www.researchgate.net/project/DOMIPEX-Carbon-uptake-in-headwater-streams-the-first-collaborativeexperiment-among-young-researchers

iv https://freshproject-eurorun.jimdo.com/eurorun/project-eurorun/

${ }^{v}$ https://freshproject-urbanalgae.jimdo.com/teams/

${ }^{v i}$ https://www.researchgate.net/project/A-timely-look-at-effects-of-agriculture-on-fluvial-dissolved-organicmatter-the-role-of-hydrology-AGRHYDROM
}

Page 3 of 11 
60 in the field methods, and teaching basic data analyses. For both projects, an extensive field

61 guide was developed. Teams pre-processed the data collected, and project coordinators

62 oversaw overall data quality control.

63 The projects covered a wide geographical area with a relatively small budget of $4500 €$ for

64

65

66

67

68

69

70

71

DOMIPEX and $8000 €$ for EuroRun. The opportunity of inviting a great number of ECRs to contribute compensated for the small economic budget of the projects. The networking among young peers took place at local or regional (i.e. micro-networking) and at continental spatial scales (i.e. macro-networking) (Fig. 1a). Teams of several ECRs at geographically close institutions jointly executed the experimental work in one location, offering a great opportunity for micro-networking. Subsequently, as part of the larger collaborative project, these teams interacted with other teams in different geographical areas, which expanded the micronetworking to macro-networking opportunities.

The teams within these two collaborative projects were either gender-balanced or had a higher proportion of female scientists ( 33 male and 49 female scientists in total; Fig. 1b). While these ratios reflect the ones existing among researchers at early stages of academic careers, they become unbalanced at later stages [8]. Other socioeconomic or racial indicators have not been specifically addressed here. However, the cultural diversity of DOMIPEX participants might have been lower compared to the EuroRun project because of constrained geographical background of the participants (AIL based in the Iberian Peninsula) than in EuroRun project (EFFS operating in Europe) (Fig. 1a). Due to the combination of these facts, we suggest that this type of project could promote the advancement of more women in their academic careers (see Career 
81 development section) and foster a more diverse scientific community, thus enhancing scientific quality, and supporting scientific careers [9].

\section{Scientific questions addressed by collaborative projects - Large trans-disciplinary} scientific societies often promote initiatives for the development of ECRs (e.g. mentoring schemes, social gatherings during conferences, etc.). However, these initiatives do not specifically focus on integrating career development with the resolution of scientific questions. Collaborative, geographically distributed projects offer the opportunity to answer large-scale research questions using innovative approaches and a structure that entirely fits with an ECR network. The distribution of teams across research institutions and countries allows a broad spatial coverage, which is essential for unraveling global environmental questions, such as the impacts of climate change on ecosystems. This might go beyond the limits of individual projects, as results can potentially be expanded to other environments [1]. Collaborative projects led by ECRs provide an intermediate between "common" scientific projects led by senior researchers with research grants and citizen science projects, where people with no scientific funding or background are involved [10]. Furthermore, because these projects bring together ECRs with no funding (e.g. for consumables and equipment acquisition, travel, organization of meetings and workshops, etc.) and diverse scientific backgrounds, the development of low-cost, simple and standardized protocols is essential. These protocols are subsequently available not only to the scientific community but can also be useful for future citizen science projects.

Career development-ECRs are especially faced with the "publish or perish" pressure and skills such as communication, idea development, project management, and funding acquisition are often neglected in the formative stages of their career $[3,11]$. Collaborative project calls 
targeted at ECRs may help to develop these neglected skills and to increase early career achievements, ensuring higher probability of future research success [12]. Consequently, the more opportunities for funding and participation in collaborative efforts that are offered to ECRs, the more likely that they have positive experiences with funding acquisition, promote our research, and continue a career in science. From an early stage, scientists are confronted with ethical challenges linked to extended teams, such as co-authorship [13] and data management [14]. Multiple tools are available at present to establish data management plans (e.g. DMPTool) and authorship attribution (e.g. CRediT system) at the initial stages of a project [15]. The participation in collaborative projects exposes the ECR to these ethical aspects early on and allows them the opportunity to address these among ourselves.

Beyond the academic domain, coping with complex environmental challenges, such as the effect of the introduction of invasive species or the causes of eutrophication, requires multidisciplinary partnerships among scientists and stakeholders from diverse backgrounds.

Translational ecology focuses on improving the connection between stakeholders and environmental scientists, for example by allowing for constant communication between the two. Translational ecology requires strong collaboration and communication skills, which are not typically included in traditional graduate training programs [11]. Collaborative projects targeted at ECRs foster the development of cooperative skills such as adaptive experiential learning, communication, leadership, and conflict resolution. We believe this can also lead to innovative solutions in team organization and task management. Collaborative projects for ECRs should thus be perceived also as instruments to nurture professional career development outside academia. 
many beneficial aspects that boost researchers' careers in the early stages. Based on the survey among the participants of DOMIPEX [5] and EuroRun, the main highlights identified were learning new concepts and methods as well as network development (Fig. 2a). Despite of having a similar management strategy, the participants in DOMIPEX valued the networking development more than in EuroRun. While the participants in EuroRun valued learning new concepts and methods more positively compared to DOMIPEX. The topics and applied methods of the two projects DOMIPEX and EuroRun defined the budget allocation, generating differences that might have strongly influenced the main benefits for the participants. For example, while DOMIPEX did not have a limited number of teams, the amount of available equipment limited the number of teams participating in EuroRun. The interaction among teams as well as constraints in project design (for EuroRun) were identified as the major challenges (Fig. 2b). While the interaction among teams was identified as a challenge in both projects to a similar extent, more than double the proportion of the participants named constraints in project design in EuroRun compared to DOMIPEX. This is most likely derived from the differences in design discussed above. Additional communication formats, such as online meetings, and a more horizontal share of project tasks (increasing individual involvement) should be implemented without increasing budget costs. Participants also suggested that project design should move towards the use of methodological tools that do not limit the number of participants.

\section{Based on the micro- and macro-networking and the high number of teams that} participated in both initiatives (Fig. 1), we postulate that these pioneer initiatives have already had a strong impact on the relationships between the ECRs of the societies that launched the 
calls. We also believe that projects targeting ECRs are likely to change the European aquatic sciences community structure in the medium to long term. Such experiences can lead to

149 stronger collaboration and networking opportunities among scientists throughout their careers,

150 which will subsequently increase scientific quality [2]. We would like to encourage senior

151 scientists supervising ECRs to continue to support such initiatives by giving their students the

152 opportunity and freedom to participate in collaborative projects. More calls designed for ECRs

153 in various scientific disciplines will foster a greater networking capacity and a more diverse and

154 integrative future for the scientific community.

156 Acknowledgments

157 We would like to thank the Iberian Association of Limnology (AIL) and the European Federation 158 for Freshwater Sciences (EFFS) for initiating the projects. AP has received support from the 159 Ramón Areces Foundation postgraduate studies program. NC held a Beatriu de Pinós grant 160 (BP2016-00215). We further acknowledge the contributions of all participants of the two 161 collaborative projects DOMIPEX and EuroRun and Laura E. Coulson for language editing. Finally, 162 we thank the reviewers Núria Cid, J. Aaron Hogan, an anonymous reviewer, and the editor, 163 Andrea E. A. Stephens, for their valuable comments which improved the paper. 
Figure captions

165 Figure 1. Geographical distribution of involved research institutions in the two pioneer early

166 career collaborative projects (the colors correspond to individual teams) (a), and the gender

167 distribution of these two projects (yellow: female, green: male) (b).

168

169 Figure 2.Results of a survey to the participants performed at the end of the collaborative

170 projects DOMIPEX (blue) and EuroRun (red) showing the highlights (a; 21 respondents for

171 DOMIPEX and 43 for EuroRun) and challenges ( $b$; 28 respondents for DOMIPEX and 43 for

172 EuroRun). The percentages were calculated for the total number of survey respondents, only

173 two options per person could be selected.

174

175 
1771 Fraser, L.H. et al. (2013) Coordinated distributed experiments: An emerging tool for testing global hypotheses in ecology and environmental science. Front. Ecol. Environ. 11,

179 147-155

1802 Pyhältö, K. and Keskinen, J. (2012) Doctoral Students' Sense of Relational Agency in Their Scholarly Communities. Int. J. High. Educ. 1, 136-149

Goring, S.J. et al. (2014) Improving the culture of interdisciplinary collaboration in ecology by expanding measures of success. Front. Ecol. Environ. 12, 39-47

1844 Bravo, A.G. et al. (2018) The interplay between total mercury, methylmercury and 185 dissolved organic matter in fluvial systems: A latitudinal study across Europe. Water Res. $144,172-182$

1875 Pastor, A. et al. (2019) Early-career coordinated distributed experiments: empowerment 188 through collaboration. bioRxiv DOI: https://doi.org/10.1101/704502

1896 Catalán, N. et al. (2018) Behind the scenes : Mechanisms regulating climatic patterns of 190 dissolved organic carbon uptake in headwater streams. Global Biogeochem. Cycles DOI: 10.1029/2018GB005919

1927 Callaway, E. (2015) Young scientists go for fresh ideas. Nature 518, 283-284

1938 Sánchez-Montoya, M. del M. et al. (2016) Women in limnology in the Iberian Peninsula: biases, barriers and recommendations. Limnetica $35,61-72$

1959 Wullum, M. et al. (2017) Gender diversity leads to better science. Proc. Natl. Acad. Sci. 
19710 Gura, T. (2013) Citizen science: amateur experts. Nature 496, 259-261

19811 Schwartz, M.W. et al. (2017) Developing a translational ecology workforce. Front. Ecol.

$199 \quad$ Environ. 15, 587-596

20012 Bol, T. et al. (2018) The Matthew effect in science funding. Proc. Natl. Acad. Sci. 115,

$201 \quad 4887-4890$

20213 Brand, A. et al. (2015) Beyond authorship: attribution, contribution, collaboration, and 203 credit. Learn. Publ. 28, 151-155

20414 Goodman, A. et al. (2014) Ten Simple Rules for the Care and Feeding of Scientific Data. 205 Plos Comput. Biol. 10,

20615 Frassl, M.A. et al. (2018) Ten simple rules for collaboratively writing a multi-authored 207 paper. Plos Comput. Biol. DOI: 10.1371/journal.pcbi.1006508

208 209 210 


\section{a) $\square$ DOMIPEX O EuroRun $\diamond$ Both}

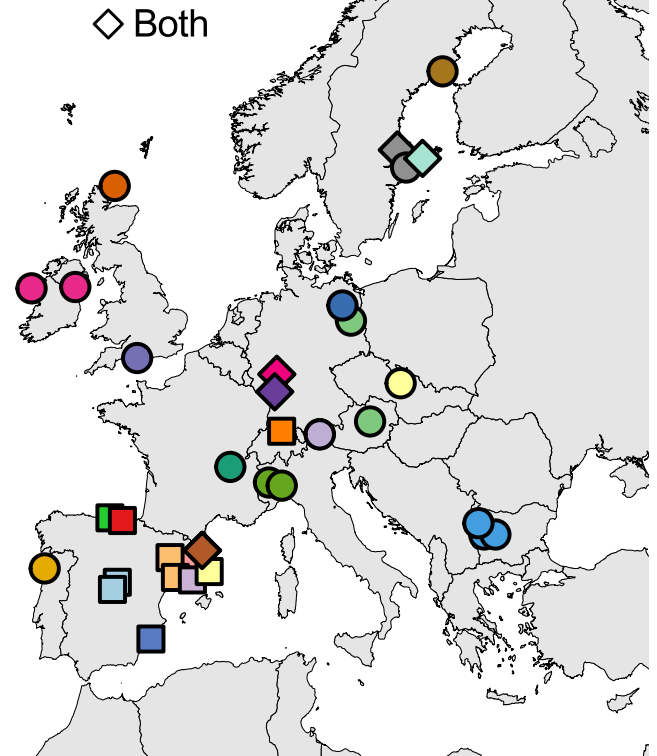

b) Group size

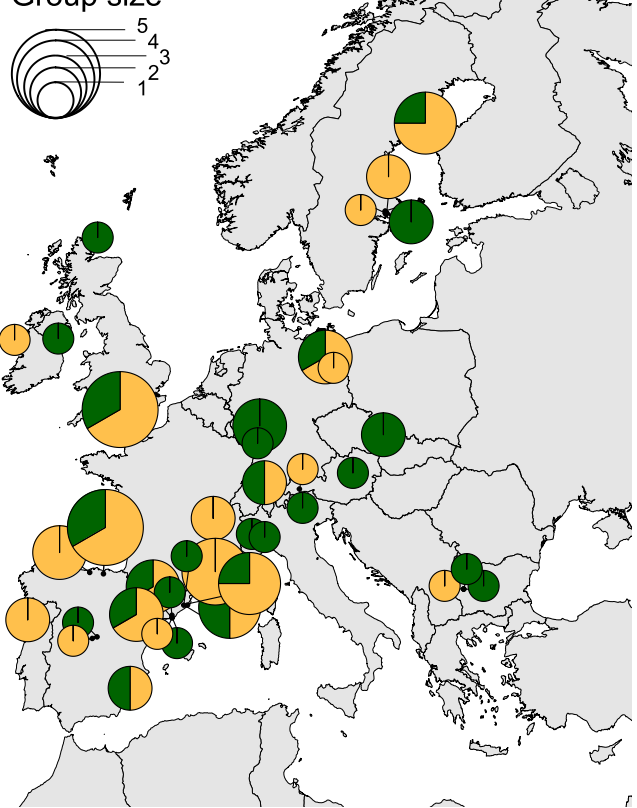


